\title{
Abbreviations of editions and works of reference
}

Classical authors are abbreviated as in LSJ, journals as in the Année Philologique. All translations are my own. For the Lives of Homer I have used my own texts and translations (Bassino 2013a). All websites were last accessed on 28 June 2018.

Arnim = Arnim, I. von (ed.) (1905), Stoicorum veterum fragmenta, vol. 1. Stuttgart.

Bernabé = Bernabé, A. (ed.) (1987), Poetae Epici Graeci: testimonia et fragmenta, vol. 1. Leipzig.

Boissonade = Boissonade, J. F. (ed.) (1851), Tzetzae Allegoriae Iliadis. Paris.

$C G F P=$ Austin, C. (ed.) (1973), Comicorum Graecorum fragmenta in papyris reperta. Berlin.

Cohoon = Cohoon, J. W. (ed.) (1932), Dio Chrysostom, vol. 1. Dio Chrysostom, I, Discourses 1-11. London.

Colonna = Colonna, A. (ed.) (1953), 'I Prolegomeni ad Esiodo e la Vita esiodea di G. Tzetzes’, BollClass 2: 27-39.

Couvreur = Couvreur, P. (ed.) (1901), Hermeias von Alexandrien. In Platonis Phaedrum scholia. Paris.

Cribiore = Cribiore, R. (1996), Writing, Teachers, and Students in Graeco-Roman Egypt. Atlanta.

Davies = Davies, M. (ed.) (1988), Epicorum Graecorum fragmenta . Göttingen .

De Lannoy = De Lannoy, L. (ed.) (1977), Flavii Philostrati Heroicus. Leipzig.

DK = Diels, H., and Kranz, W. (eds) (1961, 10 ${ }^{\text {th }}$ ed.), Die Fragmente der Vorsokratiker. Berlin.

Downey = Downey, G., et al. (eds.) (1971), Themistii Orationes quae supersunt, vol. 2. Leipzig.

Drachmann = Drachmann, A. B. (ed.) (1927), Scholia vetera in Pindari carmina, vol. 3. Leipzig.

FGrHist = Jacoby, F. (ed.) (1923- ), Die Fragmente der Griechischen Historiker. Berlin.

Fontenrose = Fontenrose, J. E. (1978), The Delphic Oracle: Its Responses and Operations, with a Catalogue of Responses. Berkeley.

Fowler = Fowler, R. L. (ed.) (2000), Early Greek Mythography, vol. 1. Oxford.

Förster = Förster, R. (ed.) (1909), Libanius. Opera, vol. 5. Leipzig.

Funaioli = Funaioli, H. (ed.) (1907), Grammaticae Romanae fragmenta, vol. 1. Leipzig.

Gaisford = Gaisford, Th. (ed.) (1823), Poetae minores Graeci, vol. 2. Leipzig.

Hubert $=$ Hubert, C. (ed.) (1971 (1938)), Plutarchi Moralia, vol. 4. Leipzig. 
$I D=$ Dürrbach, F. (ed.) (1929), Inscriptions de Délos: Comptes des Hiéropes (Nos. 372-498). Paris.

$I G=(1873-)$, Inscriptiones Graecae. Berlin.

Keaney = Keaney, J. J. (ed.) (1991), Harpocration: Lexeis of the Ten Orators. Amsterdam.

Kindstrand = Kindstrand, J. F. (ed.) (1979), Isaac Porphyrogenitus. Praefatio in Homerum. Uppsala.

LDAB = Leuven Database of Ancient Books. Online resource: http://www.trismegistos.org/ldab/

LfrgE = Snell, B., et al (eds) (1955-2010), Lexikon des frühgriechischen Epos . Göttingen.

LIMC = Kahil, L., et al. (eds) (1994), Lexicon iconographicum mythologiae classicae, vol. 7.1. Zürich.

LSJ = Liddell, H. G., Scott, R., and Jones, H. (eds) (1940, $9^{\text {th }}$ ed.), A Greek-English Lexicon. Oxford.

Macleod = Macleod, M. D. (ed.) (1972), Luciani Opera, vol. 1. Oxford.

Matthews = Matthews, V. J. (ed.) (1996), Antimachus of Colophon, Text and Commentary. Leiden.

Most $=$ Most, G. (ed.) (2006), Hesiod. Theogony. Works and Days. Testimonia . Cambridge (MA).

$\mathrm{MP}^{3}=$ Mertens-Pack 3 online database, online resource: http://cip193.philo.ulg. ac.be/Cedopal/MP3/dbsearch.aspx

MW = Merkelbach, R., and West, M. L. (eds) (1967), Fragmenta Hesiodea. Oxford.

Paton = Paton, W. R., et al. (eds) (1974 (1925)). Plutarchi Moralia, vol. 1. Leipzig.

PCG = Kassel, R., and Austin, C. (eds) (1995), Poetae Comici Graeci, vol. 8. Berlin.

Pertusi = Pertusi, A. (ed.) (1955), Scholia vetera in Hesiodi Opera et Dies. Milan.

Powell = Powell, J. U. (ed.) (1925), Collectanea Alexandrina: reliquiae minores poetarum Graecorum aetatis Ptolemaicae, 323-146 A.C.: epicorum, elegiacorum, lyricorum, ethicorum. Oxford.

Rose = Rose, V. (ed.) (1886), Aristotelis qui ferebantur librorum fragmenta . Leipzig.

Sandbach = Sandbach, F. H. (ed.) (1969), Plutarch's Moralia XV. Fragments . Cambridge (MA).

Scheer $=$ Sheer, E. (ed.) (1908), Scholia in Lycophronem, in Lycophronis Alexandra, vol. 2. Berlin.

Schneider = Schneider, O. (ed.) (1856), Nicandrea: Theriaca et Alexipharmaca . Leipzig. 
SEG $=$ Gieben, J. C., et al. (eds) (1923- ), Supplementum Epigraphicum Graecum. Amsterdam.

SGDI = Collitz, H., et al. (eds) (1884-1915), Sammlung der griechischen Dialekt-Inschriften. Göttingen.

SM = Snell, B., and Maehler, H. (eds) (1987-89), Pindari carmina cum fragmentis, 2 vols. Leipzig.

Suppl. Hell. = Lloyd-Jones, P. H. (ed.) (1983), Supplementum Hellenisticum. Berlin.

Valk, M. van der = Valk, M. van der (ed.) (1971-87), Eustathii Archiepiscopi Thessalonicensis ad Homeri Iliadem pertinentes, 4 vols. Leiden.

Voigt = Voigt, E. M. (ed.) (1971), Sappho et Alcaeus. Amsterdam.

Wehrli = Wehrli, F. (ed.) (1969, $2^{\text {nd }}$ ed.). Die Schule des Aristoteles, vol. 9. Basel.

West $=$ West, M. L. (ed.) (1989-92, $2^{\text {nd }}$ ed.), Iambi et elegi Graeci, 2 vols. Oxford.

Winiarczyk = Winiarczyk, M. (ed.) (1991), Euhemeri Messenii reliquiae. Stuttgart and Leipzig.

Wyss = Wyss, B. (ed.) (1936), Antimachi Colofonii reliquiae. Berlin. 
